\title{
Endoscopic sedation practices of Greek gastroenterologists: a nationwide survey
}

\section{Adonis A. Protopapas ${ }^{a}$, Evangelos Stournaras ${ }^{\mathrm{a}, \mathrm{b}}$, Georgios Neokosmidis ${ }^{\mathrm{a}}$, Dimitrios Stogiannou ${ }^{\mathrm{a}}$, Athanasios Filippidis ${ }^{a}$, Andreas N. Protopapas ${ }^{a}$}

Aristotle University of Thessaloniki, AHEPA Hospital, Thessaloniki, Greece; Cambridge University Hospitals NHS

Foundation Trust, Cambridge, UK

\section{Abstract}

${ }^{a}$ First Propaedeutic Department of Internal Medicine, Aristotle University of Thessaloniki, AHEPA Hospital, Thessaloniki, Greece (Adonis A. Protopapas, Evangelos Stournaras, Georgios Neokosmidis, Dimitrios Stogiannou, Athanasios Filippidis, Andreas N. Protopapas); ${ }^{\mathrm{b} C a m b r i d g e}$ University Hospitals NHS Foundation Trust, Cambridge, United Kingdom (Evangelos Stournaras)

\section{Conflict of Interest: None}

Correspondence to: Adonis A. Protopapas, Resident of Gastroenterology, $1^{\text {st }}$ Propaedeutic Department of Internal Medicine, Aristotle University of Thessaloniki, AHEPA Hospital, Thessaloniki, Greece, e-mail: adoprot@hotmail.com

Received 29 January 2020; accepted 21 April 2020; published online 15 May 2020

DOI: https://doi.org/10.20524/aog.2020.0494

\section{Introduction}

Gastrointestinal endoscopy practices are rapidly evolving, addressing a significant number of previously unmet needs. This evolution goes hand in hand with the evolution of endoscopic sedation practices, with sedation being employed to minimize patient discomfort and improve the quality of the procedure [1]. However, there is an ongoing debate regarding the possible complications of sedation and whether non-anesthesiologists should be responsible for the administration of sedation in gastrointestinal endoscopy. Although the use by non-anesthesiologists of regimens that induce moderate sedation, such as midazolam and fentanyl, has been accepted by anesthesiological societies [2], the 
debate still rages regarding the administration of propofol, a regimen that induces deep sedation. There have been a significant number of studies addressing this debate, with the vast majority of them concluding that propofol can be safely provided by non-anesthesiologists, without differences in safety outcomes [3-6]. This evidence, along with evidence that propofol is superior in terms of efficacy (shorter recovery time, better patient and physician satisfaction) compared to other endoscopic regimens [7-9], leads to arguments that propofol may indeed be the optimal method of sedation for gastrointestinal endoscopy [10]. However, contradictions between statements from gastroenterological [11,12] and anesthesiological $[13,14]$ organizations have led to different patterns regarding the non-anesthesiologist administration of propofol (NAAP) among countries worldwide. For instance, in the USA NAAP is essentially prohibited, and propofol use is restricted to anesthesiologists, taking account of the FDA warning stating that propofol "should be administered only by persons trained in the administration of general anesthesia and not involved in the conduct of the surgical/diagnostic procedure" [15]. In contrast, in a survey in 2011, as many as $98 \%$ of German gastroenterologists were found to use propofolbased regimens for sedation [16].

There have been many surveys regarding the use of sedation for gastrointestinal endoscopy, with significant differences being observed depending on the country where each survey was conducted [16-20]. However, there has been only one study in Greece, conducted in 2007 [21]. While relatively recent, this study was carried out before the development of guidelines that encourage the use of NAAP. This is the knowledge gap that our survey was designed to fill. In addition, the absence of a legal framework for the non-anesthesiologist administration of any kind of intravenous sedation in our country motivated us to highlight the sedation practices of gastroenterologists, to promote judicial adjustments. To sum up, the aim of this study was to document the sedation practices of Greek gastroenterologists, in order to identify differences from other countries and to help highlight areas of possible improvement.

\section{Materials and methods}

\section{Study characteristics}

The study was conducted using an online questionnaire sent to all specialist gastroenterologists, members of the Hellenic Society of Gastroenterology (HSG). The participants were contacted in an e-mail from the study coordinator (ANP), where the purpose of the questionnaire was explained and a link for the completion of the online questionnaire was provided. The answers to the questionnaire were anonymous. The survey was conducted on 2 separate occasions, December 2015 and June 2018, to identify changing patterns. The same survey instrument was used in both instances. A reminder e-mail to increase the response rate was sent in both instances, in January 2016 and July 2018, respectively. The questionnaire was sent to 509 and 547 gastroenterologists, respectively.

\section{Questionnaire}

The questionnaire was comprised of 39 questions, 14 of which were related to the practice of endoscopic retrograde cholangiopancreatography (ERCP) and endoscopic ultrasound (EUS). The questions were used to determine demographic and personal practice data, use of sedation, type of sedation used and motivation behind its use, collaboration with anesthesiologists, safety measures, satisfaction with sedation, and personal preference regarding sedative agent if the responder were to undergo endoscopy. In an effort to thoroughly evaluate the physician's practices, many questions were organized in a Likert scale of 4 possible answers: never/almost never (0-25\% of the time), seldom (25-50\% of the time), usually (50-75\% of the time), and always/almost always (75-100\% of the time). Questions regarding sedation type and the use of sedation antagonists were available only to those administering sedation (even seldom), while questions regarding the reason for using/ not using propofol were available according to whether the physician used propofol. The questionnaire is provided in the supplementary material, with the original (Greek) version and a version translated into English.

\section{Statistical analysis}

All statistical analysis was performed using the SPSS 25.0 software package (IBM Corp. (C)). The KolmogorovSmirnov test was used to determine if the data of continuous variables were normally distributed. We used Student's $t$-test and analysis of variance to compare numeric values with normal distribution, and the MannWhitney or Kruskal-Wallis tests to compare numeric values without normal distribution. The chi-square test was used to compare categorical values and McNemar's test was used to determine if there were differences in a dichotomous dependent variable between the 2 related groups (specifically, comparing the use of propofol with the preference of being sedated with propofol). Values are described as mean \pm standard deviation for normal variables and as median (range) for non-normal variables. $\mathrm{P}$-values $<0.05$ were considered as statistically significant.

\section{Results}

\section{Demographics and practice patterns}

Questionnaires were answered by 195 and 258 gastroenterologists, representing response rates of $38.3 \%$ and $47.1 \%$ for 2015 and 2018 , respectively. The responders were mainly men and physicians working in the private sector, while the area of practice was divided between the 2 major cities (Athens and Thessaloniki, accounting for 50-60\% responses in both periods) and the rest of the country (40-50\%). Demographic data from each period are shown in Table 1. 
Regarding the volume of endoscopies performed, gastroenterologists performed a median of 10 (0-30) esophagogastroduodenoscopies (EGDs) and 10 (30) colonoscopies per week in the first period, while they performed a median of 10 (0-40) EGDs and 10 (0-30) colonoscopies per week in the second period. When categorized based on the volume of endoscopies, the number of physicians performing $<10$ EGDs per week was $102(52.3 \%)$ and 136 (52.7\%), the number performing 10-20 was $57(29.2 \%)$ and $87(33.7 \%)$, and the number performing $>20$ was $36(18.5 \%)$ and 35 (13.6\%), for each period respectively. Regarding colonoscopies, the number of physicians performing $<10$ per week was $100(51.3 \%)$ and 148 (57.4\%), the number performing $10-20$ was $62(31.8 \%)$ and $79(30.6 \%)$, and the number performing $>20$ was $33(16.9 \%)$ and 31 (12\%), for each period respectively.

The majority of physicians answered in both periods that they usually or always supply oxygen to their patients during endoscopy, though there was an increase in the routine use of supplementary oxygen in the second period $(\mathrm{P}=0.015)$. With regard to the vital signs evaluated during endoscopy, $>95 \%$ of gastroenterologists reported the monitoring of oxygen saturation and heart rate in both periods, and around $40 \%$ reported the monitoring of arterial pressure. Electrocardiography (ECG) and capnography were reported by a relatively small number of physicians. Finally, regarding the presence of an anesthesiologist during endoscopy, the vast majority ( $>2 / 3$ of the answers) answered that they never/almost never collaborate with an anesthesiologist during endoscopy. In the second period,

Table 1 Descriptive statistics of gastroenterologists who participated in the study in each period

\begin{tabular}{lcc}
\hline Period & 2015 & 2018 \\
\hline Participants & 195 & 258 \\
Response rate & $38.3 \%$ & $47.1 \%$ \\
Sex & & \\
\multicolumn{1}{l}{ Male } & $165(84.6 \%)$ & $204(79.1 \%)$ \\
Female & $30(15.4 \%)$ & $54(20.9 \%)$ \\
Occupation & & \\
Private sector & $126(64.6 \%)$ & $159(61.6 \%)$ \\
Public sector & $54(27.7 \%)$ & $83(32.2 \%)$ \\
Academic & $15(7.7 \%)$ & $16(6.2 \%)$ \\
\hline Area & & $118(45.7 \%)$ \\
Athens & $73(37.4 \%)$ & $28(10.9 \%)$ \\
Thessaloniki & $36(18.5 \%)$ & $84(32.6 \%)$ \\
City $>50,000$ & $67(34.4 \%)$ & $28(10.9 \%)$ \\
City <50,000 & $19(9.7 \%)$ & $48(32-70)$ \\
\hline Age & $48(36-69)$ & $12(0-38)$ \\
\hline Years of practice & $13(1-37)$ & \\
\hline Continuous variables are expressed as median (range) &
\end{tabular}

Continuous variables are expressed as median (range) physicians working in the private sector were more likely to collaborate with an anesthesiologist $(\mathrm{P}=0.001)$, unlike the first period $(\mathrm{P}=0.069)$. Detailed answers are outlined in Table 2.

\section{Use of sedation}

Sedation use was reported by most physicians, with $132(68.2 \%)$ and $193(74.8 \%)$ reporting systematical use of sedation ("always/almost always" or "usually") in the first and second periods, respectively. However, a significant number of physicians reported that they do not use sedation ("never/ almost never") in both periods (25.1\% in 2015 and $16.7 \%$ in 2018). Physicians with private practices were more likely to use sedation than physicians of the public sector or academics ( $\mathrm{P}=0.013$ for 2015 and 0.036 for 2018). Interestingly, there appears to be an increase in the use of sedation during these 2.5 years $(\mathrm{P}=0.01)$, highlighted by the increase in the physicians who "always" use sedation, from $47.2 \%$ to $61.2 \%$. Sedation use correlated significantly with the physician's age $(\mathrm{P}=0.005)$ and years of practice $(\mathrm{P}=0.002)$ in 2015 , with younger and less experienced physicians more likely to use sedation. However, this correlation was not maintained in the second period $(\mathrm{P}=0.533$ and 0.509$)$. With regard to the use of agents that act as antagonists to sedation regimens in order to reverse sedation, most physicians reported frequent use of such a drug in both periods. Responses regarding sedation use and antagonist use are shown in Table 3.

Table 2 Oxygen supply, vital signs and collaboration with anesthesiologists during endoscopy

\begin{tabular}{lccc}
\hline Period & 2015 & 2018 & P-value \\
\hline Oxygen supply & $\mathrm{n}=195$ & $\mathrm{n}=258$ & 0.015 \\
Never/almost never & $31(15.9 \%)$ & $26(10.1 \%)$ & \\
Seldom & $34(17.4 \%)$ & $43(16.7 \%)$ & \\
Usually & $43(22.1 \%)$ & $44(17.1 \%)$ & \\
Always/almost & $87(44.6 \%)$ & $145(56.2 \%)$ & \\
always & & & \\
Vital signs & $\mathrm{n}=195$ & $\mathrm{n}=258$ & $>0.05$ \\
Oxygen saturation & $189(96.9 \%)$ & $252(97.7 \%)$ & \\
Heart rate & $188(96.4 \%)$ & $254(98.4 \%)$ & \\
Arterial pressure & $78(40 \%)$ & $101(39.1 \%)$ & \\
Electrocardiogram & $22(11.3 \%)$ & $18(7 \%)$ & \\
Capnography & $4(2.1 \%)$ & $8(3.1 \%)$ & \\
Anesthesiologist & $\mathrm{n}=195$ & $\mathrm{n}=258$ & $>0.05$ \\
Never/almost never & $138(70.8 \%)$ & $176(68.2 \%)$ & \\
Seldom & $34(17.4 \%)$ & $47(18.2 \%)$ & \\
Usually & $12(6.2 \%)$ & $11(4.3 \%)$ & \\
Always/almost & $11(5.6 \%)$ & $24(9.3 \%)$ & \\
always & & & \\
\hline
\end{tabular}




\section{Sedation regimens}

The preferred combinations of regimens used in EGDs and colonoscopies are shown in Fig. 1. Monotherapy with midazolam was the most preferred type of sedation for EGDs in both periods ( $50 \%$ and $47.4 \%$, respectively). Midazolam was by far the most popular drug (either alone or in combination with other regimens) in EGDs, used by $91.9 \%$ and $93 \%$ of physicians, respectively. Regarding colonoscopies, many types of sedation were frequently used. The most frequently reported combination was midazolam with fentanyl $(24.6 \%$ and $28.4 \%$, respectively), followed by midazolam monotherapy (21.9\% and 22.8\%, respectively). Again, midazolam was by far the most popular drug, used by $89.7 \%$ and $91.6 \%$ of the gastroenterologists using sedation in each period, respectively.

Table 3 Use of sedation and sedation antagonist by gastroenterologists

\begin{tabular}{lccc}
\hline Period & 2015 & 2018 & P-value \\
\hline Use of sedation & $\mathrm{n}=195$ & $\mathrm{n}=258$ & 0.01 \\
Never/almost never & $49(25.1 \%)$ & $43(20.5 \%)$ & \\
Seldom & $14(7.2 \%)$ & $22(8.5 \%)$ & \\
Usually & $40(20.5 \%)$ & $35(13.6 \%)$ & \\
Always/almost always & $92(47.2 \%)$ & $158(61.2 \%)$ & \\
Use of sedation antagonist & $\mathrm{n}=146$ & $\mathrm{n}=215$ & $>0.05$ \\
Never/almost never & $27(18.5 \%)$ & $44(20.5 \%)$ & \\
Seldom & $27(18.5 \%)$ & $46(21.4 \%)$ & \\
Usually & $28(19.2 \%)$ & $29(13.5 \%)$ & \\
Always/almost always & $64(43.8 \%)$ & $96(44.7 \%)$ & \\
\hline
\end{tabular}

\section{Propofol}

Propofol was used as a part of the preferred regimen by $45(30.8 \%)$ and 58 (27\%) physicians who used sedation in each period, respectively. Among those physicians, most cited "better patient cooperation" as the chief reason behind the use of propofol (66.7\% and $53.4 \%$, respectively), while some cited "increase in quality of endoscopy" (28.9\% and $31 \%$, respectively). Among physicians who did not routinely use propofol $(69.2 \%$ and $73 \%$ of those using sedation, respectively), chief reasons were medicolegal issues $(34.7 \%$ and $36.3 \%$, respectively), inadequate training $(27.7 \%$ and $28 \%$, respectively) and the possibility of cardiopulmonary complications $(24.8 \%$ and $21.7 \%$, respectively). Physicians using propofol were more likely to collaborate with an anesthesiologist $(\mathrm{P}<0.001$ for both periods), although only a small percentage reported systematic (usually or always) collaboration (33.3\% and $39.7 \%$, respectively). Physicians of the private sector were more likely to use propofol in both periods $(\mathrm{P}=0.016$ and $\mathrm{P}=0.001$, respectively). Finally, propofol use was not significantly influenced by the age or years of practice of the responding physicians in either period.

\section{Safety equipment}

Almost all the gastroenterologists reported the presence of an oxygen supply in their practice (98\% and $98.8 \%$, respectively). The vast majority of the responders had access to laryngeal masks/oropharyngeal airways (80\% and $79.8 \%$, respectively) and drug regimens for cardiopulmonary resuscitation $(81.5 \%$ and $82.6 \%$, respectively), while almost

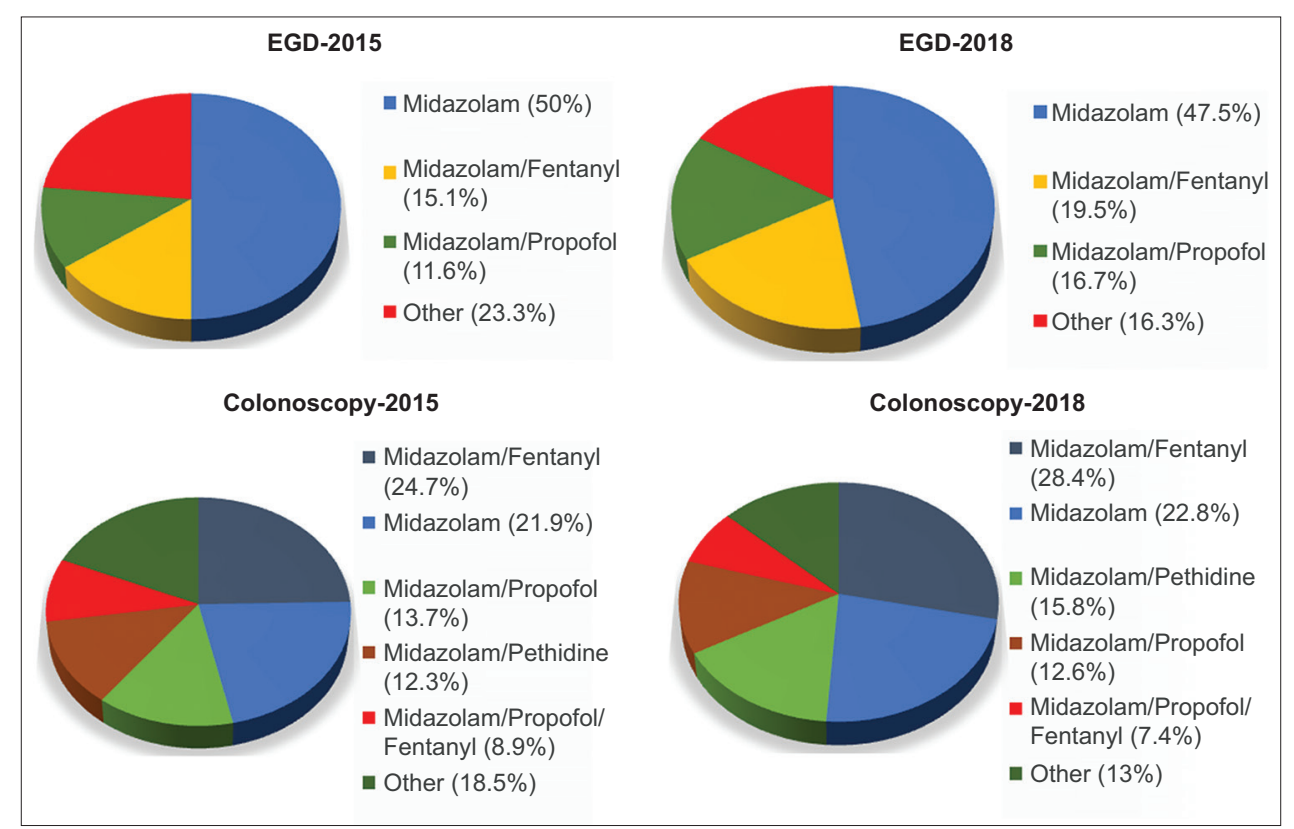

Figure 1 Drug regimens used by gastroenterologists for sedation during esophagogastroduodenoscopy (EGD) or colonoscopy 
half of the physicians had access to a defibrillator ( $44.6 \%$ and $47.7 \%$, respectively) and endotracheal intubation equipment (47.2\% and $48.8 \%$, respectively). These numbers were greater in the case of physicians using propofol: laryngeal masks/ oropharyngeal airways were reported to be available to $88.9 \%$ and $94.8 \%$, drug regimens for cardiopulmonary resuscitation to $80 \%$ and $82.8 \%$, a defibrillator to $46.7 \%$ and $56.9 \%$, and endotracheal intubation to $64.4 \%$ and $75.9 \%$ of the physicians, for each period respectively.

\section{Physician satisfaction}

When asked to rank their satisfaction with the sedation regimen they use on a scale from 1 to 10 , the physicians scored median scores of 8 (1-10) and 8 (3-10) in each respective period. The majority of the physicians achieved a score of $\geq 8(65.6 \%$ and $67.1 \%$, respectively). In the second period $(\mathrm{P}<0.001)$, albeit not in the first $(\mathrm{P}=0.304)$, physicians using sedation scored significantly better than those who did not. In both periods, propofol use was associated with better satisfaction than the use of other types of sedation $(\mathrm{P}<0.001$ for both periods) and no sedation ( $\mathrm{P}<0.001$ for both periods). Furthermore, monotherapy with propofol would be the most selected regimen by the physicians, should they undergo gastrointestinal endoscopy (21\% and $22.9 \%$, respectively), while also being among the drugs of the preferred combination in the majority of cases $(53.8 \%$ and $53.1 \%$, respectively). There was a significant difference between the preference of a physician for the sedation of his patients and his personal sedation, with many physicians preferring propofol for their personal sedation, although not using it as part of their practice $(\mathrm{P}<0.001$ for both periods). The preferred regimens are illustrated in Fig. 2.

\section{ERCP}

Thirty-seven (19\%) and 48 (18.6\%) physicians conducted ERCP in each period. Gastroenterologists working in the public sector were more likely to conduct ERCP $(\mathrm{P}<0.001$ for both periods). In both periods, there was a significant division between gastroenterologists regarding the presence of an anesthesiologist during ERCP, with $32.4 \%$ and $41.7 \%$ reporting never collaborating with an anesthesiologist and $32.4 \%$ and $39.6 \%$ reporting always collaborating with an anesthesiologist, respectively. The presence of an anesthesiologist during ERCP was significantly correlated with the type of practice $(\mathrm{P}=0.006$ and $\mathrm{P}<0.001$ for each period), with private-sector physicians being more likely to collaborate with an anesthesiologist. Almost all gastroenterologists always used supplementary oxygen during ERCP (94.6\% and 100\%, respectively). The monitoring of vital signs during ERCP is displayed in Table 4. Regarding sedation, the most frequent combinations were midazolam/pethidine $(32.4 \%$ and $29.2 \%$ for each period) and midazolam/fentanyl/propofol (35.1\% and $22.9 \%$ for each period). Midazolam was the most widely used drug $(89.2 \%$ and $85.4 \%$, respectively), while a significant number of physicians used propofol ( $59.5 \%$ and $52.1 \%$, respectively), fentanyl ( $40.5 \%$ and $54.2 \%$, respectively), and pethidine $(43.2 \%$ and $43.8 \%$, respectively).

Table 4 Monitoring of vital signs during endoscopic retrograde cholangiopancreatography (ERCP) and endoscopic ultrasound (EUS)

\begin{tabular}{lcc} 
Period & 2015 & 2018 \\
\hline Vital signs - ERCP & $\mathrm{n}=37$ & $\mathrm{n}=48$ \\
Oxygen saturation & $36(97.3 \%)$ & $48(100 \%)$ \\
Heart rate & $37(100 \%)$ & $48(100 \%)$ \\
Arterial pressure & $23(62.2 \%)$ & $35(72.9 \%)$ \\
Electrocardiogram & $14(37.8 \%)$ & $23(47.9 \%)$ \\
Capnography & $9(24.3 \%)$ & $9(18.8 \%)$ \\
Vital signs - EUS & $\mathrm{n}=14$ & $\mathrm{n}=20$ \\
Oxygen saturation & $14(100 \%)$ & $20(100 \%)$ \\
Heart rate & $14(100 \%)$ & $20(100 \%)$ \\
Arterial pressure & $5(35.7 \%)$ & $11(55 \%)$ \\
Electrocardiogram & $1(7.1 \%)$ & $5(25 \%)$ \\
Capnography & $0(0 \%)$ & $2(20 \%)$ \\
\hline
\end{tabular}

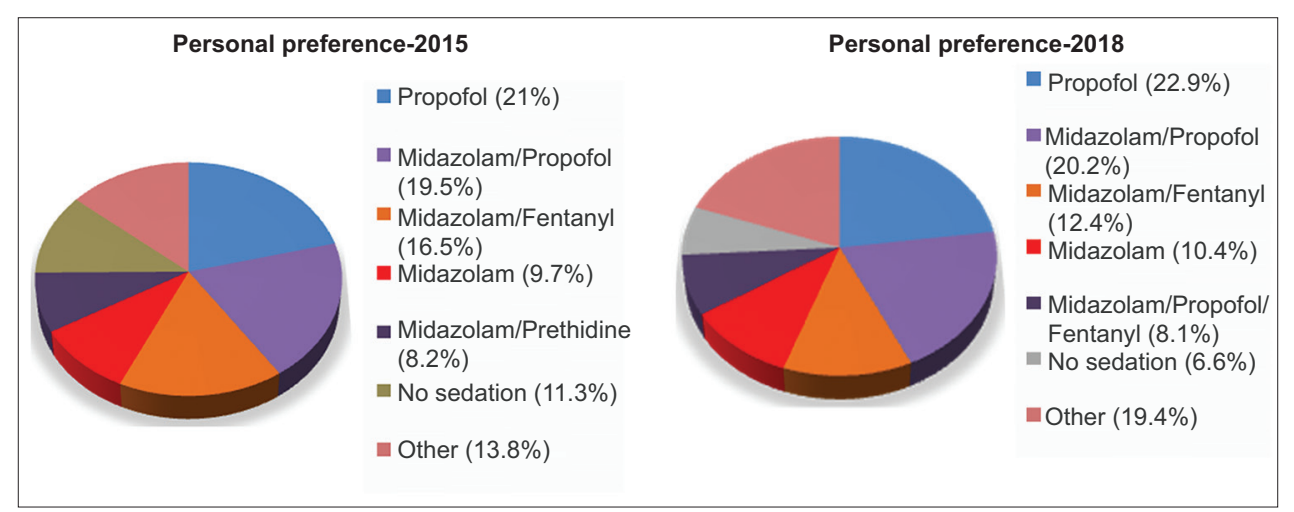

Figure 2 Personal preferences for sedation if the gastroenterologist should undergo endoscopy 


\section{EUS}

EUS was practiced by 14 (7.2\%) and 20 (7.8\%) physicians in each period. Gastroenterologists working in the public sector were more likely to conduct EUS in the second period $(\mathrm{P}=0.002)$, but not in the first $(\mathrm{P}=0.064)$. Most physicians seldom or never enlisted the help of an anesthesiologist (64.3\% and $70 \%$, respectively). The majority of physicians routinely used supplementary oxygen during the procedure $(100 \%$ and $95 \%$, respectively). The monitoring of vital signs during EUS is displayed in Table 4. Most physicians (>85\%) used a combination of substances as their preferred sedation regimen, with midazolam being used extensively (93.9\% and 100\%, respectively), followed by propofol (50\% in both periods) and fentanyl ( $50 \%$ in both periods).

\section{Discussion}

This study attempted to identify the endoscopic sedation practices of Greek gastroenterologists in 2 separate periods. Our goal was to identify changes in the practice of gastroenterologists in Greece, among the rapidly changing landscape of gastrointestinal endoscopy, while at the same time focusing on the ambiguous matter of intravenous sedation. Our study shows that intravenous sedation is common practice for most gastroenterologists in Greece, with more and more gastroenterologists routinely using sedation, as highlighted by the increase in the percentage of gastroenterologists using sedation between the 2 periods of our study. In the vast majority of cases, endoscopy is conducted without the presence of an anesthesiologist. Additionally, midazolam is by far the most used agent, with over $90 \%$ of gastroenterologists systematically administering it as monotherapy or in combination with other drugs. Propofol is not widely used, mainly because of medicolegal reasons and inadequate training. However, propofol is much more desirable for endoscopists if they were themselves to undergo gastrointestinal endoscopy. Furthermore, gastroenterologists seem to conform to safety standards regarding vital sign monitoring and the presence of safety equipment, with the vast majority of them having access to airway management devices and cardiopulmonary resuscitation drug regimens.

There have been many surveys that have delved into the matter of endoscopic sedation. These surveys display consistent similarities when it comes to the use of midazolam without the aid of an anesthesiologist and significant discrepancies regarding the person responsible for the administration of propofol. It is universally accepted that midazolam can be used without the presence of an anesthesiologist. However, depending on the country, the use of propofol without the presence of an anesthesiologist may vary from $0 \%$ to almost $100 \%$ [16-21]. Interestingly, in these surveys, propofol is the most frequently used sedation regimen in countries where it is administered mainly by non-anesthesiologists, while midazolam is the most common regimen in countries where propofol is administered mainly by anesthesiologists. Surveys from the USA, Italy and Portugal showed that propofol administration was almost exclusively directed by anesthesiologists [18-20], while surveys from Germany and Spain displayed opposite results, with propofol administration being almost entirely directed by non-anesthesiologists $[16,17]$. One key difference may be the presence of national German and Spanish guidelines that support the use of propofol by non-anesthesiologists [22,23]. More significantly, the German Society for Anesthesia and Intensive Care Medicine actively participated in the design of the national German guideline, a feat that the American (ASGE) and European (ESGE) Societies for Gastrointestinal Endoscopy have not been able to replicate $[11,12,24]$, although the European Society of Anesthesiology initially supported the use of NAAP but later retracted its support [13,14].

Our study's main differences compared to the previous study conducted in Greece are also related to the use of propofol. In the previous study, propofol was referred to only as an option for selected cases, with up to $28 \%$ of physicians reporting its use [21]. Although this percentage is similar to ours, in our study, physicians reported the use of propofol as part of their preferred regimen for endoscopy. Furthermore, the increase in propofol use has coincided with a decrease in the instances where propofol administration is directed by an anesthesiologist. This can be attributed to the added difficulty of collaborating with an anesthesiologist on a regular basis, compared to enlisting his/her aid for selected cases. These differences were to be expected, considering that there were no guidelines in place for the use of NAAP at the time of the previous study. Furthermore, physicians in our study seem to be more prepared for possible cardiopulmonary complications, with a significantly higher percentage of the physicians having access to necessary safety equipment and routinely supplying oxygen to the patient.

Our study has certain strengths and limitations. The principal advantage of our study was its demonstrated reproducibility. The results were almost identical between the 2 periods, showing that the answers accurately represent the endoscopic practices of Greek gastroenterologists. The use of a 4-point Likert scale achieves a balanced depiction of sedation practices, demonstrating that endoscopic patterns change depending on patient profile and situation. A notable limitation of the study was that a significant number of gastroenterologists did not respond ( $>50 \%$ for both periods). However, the response rate was similar if not superior to other similar surveys [16-21]. The chief limitations of the survey arise due to structural errors in the questionnaire: the question regarding the preferred regimen of physicians should they undergo endoscopy could have been divided into 2 questions ( 1 for EGD and 1 for colonoscopy) and followed by a question regarding their preference regarding the person responsible for administering the sedation. The latter part was undoubtedly influenced by the fact that anesthesiologistdirected sedation is not common practice in Greece; thus, the assumption was that most physicians would consider this 
question on the basis that sedation would be administered by a non-anesthesiologist. Finally, self-reporting of personal practices always carries the risk of the subjectivity of the person being asked.

Sedation has been the backbone of the significant increase in endoscopic procedures worldwide. Sedation has been reported to be one of the most significant factors that influence the patient to undergo colonoscopy $[25,26]$. However, the use of sedation leads to an increase in the cost of endoscopy, an increase that is exceptionally significant if the sedation is administered by an anesthesiologist [27-30]. A study by Hassan et al estimated savings of $\$ 3.2$ billion per 10 years in the USA, if propofol administration was directed by endoscopists instead of anesthesiologists [31]. Considering that the financial cost of colonoscopy is reported to be a significant deterrent for colorectal cancer screening $[25,32,33]$, the added cost of anesthesia providers may further dissuade low-income patients from undergoing screening. These concerns are mainly associated with the administration of propofol and not midazolam, the sedation agent mainly used by Greek gastroenterologists. However, the fact that in our study the gastroenterologists using propofol were significantly more satisfied and that propofol was the most preferred regimen if the physicians were to undergo an endoscopy, in combination with the increasing use of propofol for endoscopic sedation worldwide, warrants a solution that would enable the use of propofol by nonanesthesiologists, without implications for the safety of the patient. Such a solution may be feasible with the use of a comprehensive endoscopic sedation curriculum that would ensure the proper training of non-anesthesiologists, similar to those developed by the ASGE and ESGE [34,35]. The fact that almost all the endoscopists administering propofol report having access to airway management devices shows that gastroenterologists are familiar with the safety equipment necessary for the administration of propofol. The utilization of a strict curriculum that ensures their qualification for administering sedation, monitoring patients and handling complications could be the key to establishing the safe use of NAAP in Greece.

To conclude, sedation is considered as a prerequisite for gastrointestinal endoscopy by most Greek gastroenterologists. Midazolam is by far the most widely used sedation agent, while propofol use is continuously increasing and is likely to increase more, as it achieves better physician satisfaction and is mentioned by most gastroenterologists as their preferred method of sedation should they undergo endoscopy themselves. Administration of sedation is mostly directed by gastroenterologists, even when it comes to propofol use. Furthermore, the vast majority of gastroenterologists have access to safety equipment essential for the management of airway complications. All things considered, these results show that the only legitimate obstacle preventing endoscopistdirected sedation and NAAP in Greece is the development of a strict curriculum for endoscopic sedation, with the cooperation of the anesthesiological community and the support of state legislature.

\section{Summary Box}

\section{What is already known:}

- Endoscopic sedation practices are rapidly changing worldwide

- There are significant differences in sedation practices between countries

- There is a significant increase in nonanesthesiologist administration of propofol in many countries

\section{What the new findings are:}

- Midazolam is by far the most commonly used sedative agent by Greek gastroenterologists

- Anesthesiologists are rarely involved in the administration of sedation for gastrointestinal endoscopy in Greece

- Vital sign monitoring and safety equipment are available in the vast majority of Greek endoscopic practices

- Propofol is considered as the best option by many gastroenterologists, but its use is hindered by medicolegal reasons and a lack of appropriate training

\section{References}

1. McQuaid KR, Laine L. A systematic review and meta-analysis of randomized, controlled trials of moderate sedation for routine endoscopic procedures. Gastrointest Endosc 2008;67:910-923.

2. American Society of Anesthesiologists Task Force on Sedation and Analgesia by Non-Anesthesiologists. Practice guidelines for sedation and analgesia by non-anesthesiologists. Anesthesiology 2002;96:1004-1017.

3. Rex DK, Deenadayalu VP, Eid E, et al. Endoscopist-directed administration of propofol: a worldwide safety experience. Gastroenterology 2009;137:1229-1237.

4. Friedrich K, Stremmel W, Sieg A. Endoscopist-administered propofol sedation is safe - a prospective evaluation of 10,000 patients in an outpatient practice. J Gastrointestin Liver Dis 2012;21:259-263.

5. Sieg A, Beck S, Scholl SG, et al; bng-Study-Group. Safety analysis of endoscopist-directed propofol sedation: a prospective, national multicenter study of 24441 patients in German outpatient practices. J Gastroenterol Hepatol 2014;29:517-523.

6. Vargo JJ, Niklewski PJ, Williams JL, Martin JF, Faigel DO. Patient safety during sedation by anesthesia professionals during routine upper endoscopy and colonoscopy: an analysis of 1.38 million procedures. Gastrointest Endosc 2017;85:101-108.

7. Sethi S, Wadhwa V, Thaker A, et al. Propofol versus traditional sedative agents for advanced endoscopic procedures: a metaanalysis. Dig Endosc 2014;26:515-524.

8. Wang D, Chen C, Chen J, et al. The use of propofol as a sedative agent in gastrointestinal endoscopy: a meta-analysis. PLoS One 2013;8:e53311.

9. Singh H, Poluha W, Cheung M, Choptain N, Baron KI, Taback SP. 
Propofol for sedation during colonoscopy. Cochrane Database Syst Rev 2008;(4):CD006268.

10. Stogiannou D, Protopapas A, Protopapas A, Tziomalos K. Is propofol the optimal sedative in gastrointestinal endoscopy? Acta Gastroenterol Belg 2018;81:520-524.

11. Dumonceau JM, Riphaus A, Schreiber F, et al. Non-anesthesiologist administration of propofol for gastrointestinal endoscopy: European Society of Gastrointestinal Endoscopy, European Society of Gastroenterology and Endoscopy Nurses and Associates Guideline-Updated June 2015. Endoscopy 2015;47:1175-1189.

12. Vargo JJ, Cohen LB, Rex DK, Kwo PY. Position statement: Nonanesthesiologist administration of propofol for GI endoscopy. Hepatology 2009;50:1683-1689.

13. Pelosi P; Board of the European Society of Anaesthesiology. Retraction of endorsement: European Society of Gastrointestinal Endoscopy, European Society of Gastroenterology and Endoscopy Nurses and Associates and the European Society of Anaesthesiology Guideline-non-anaesthesiologist administration of propofol for gastrointestinal endoscopy. Eur J Anaesthesiol 2012;29:208.

14. Perel A. Non-anaesthesiologists should not be allowed to administer propofol for procedural sedation: A Consensus Statement of 21 European National Societies of Anaesthesia. Eur J Anaesthesiol 2011;28:580-584.

15. Electronic Medicines Compendium (eMC) for propofol. Section: special warnings and precautions for use.

16. Riphaus A, Geist F, Wehrmann T. Endoscopic sedation and monitoring practice in Germany: re-evaluation from the first nationwide survey 3 years after the implementation of an evidence and consent based national guideline. Z Gastroenterol 2013;51:1082-1088.

17. Lucendo AJ, González-Huix F, Tenias JM, et al; Spanish Society of Digestive Diseases, Spanish Society of Digestive Endoscopy, and Spanish Association of Gastroenterology. Gastrointestinal endoscopy sedation and monitoring practices in Spain: a nationwide survey in the year 2014. Endoscopy 2015;47:383-390.

18. Ferreira AO, Torres J, Dinis-Ribeiro M, Cravo M. Endoscopic sedation and monitoring practices in Portugal: a nationwide webbased survey. Eur J Gastroenterol Hepatol 2015;27:265-270.

19. Fanti L, Agostoni M, Gemma M, et al; Italian Society of Digestive Endoscopy Sedation Commission. Sedation and monitoring for gastrointestinal endoscopy: A nationwide web survey in Italy. Dig Liver Dis 2011;43:726-730.

20. Cohen LB, Wecsler JS, Gaetano JN, et al. Endoscopic sedation in the United States: results from a nationwide survey. Am J Gastroenterol 2006;101:967-974.

21. Paspatis GA, Manolaraki MM, Tribonias G, et al. Endoscopic sedation in Greece: results from a nationwide survey for the Hellenic Foundation of gastroenterology and nutrition. Dig Liver Dis 2009;41:807-811.

22. Riphaus A, Wehrmann T, Weber B, et al. S3 Guideline: Sedation for gastrointestinal endoscopy 2008. Endoscopy 2009;41:787-815.

23. López Rosés L, Subcomité de Protocolos of the Spanish Society of Gastrointestinal Endoscopy Seed. Sedation/analgesia guidelines for endoscopy. Rev Esp Enferm Dig 2006;98:685-692.

24. Early DS, Lightdale JR, Vargo JJ $2^{\text {nd }}$, et al; ASGE Standards of Practice Committee. Guidelines for sedation and anesthesia in GI endoscopy. Gastrointest Endosc 2018;87:327-337.

25. Harewood GC, Wiersema MJ, Melton LJ $3^{\text {rd }}$. A prospective, controlled assessment of factors influencing acceptance of screening colonoscopy. Am J Gastroenterol 2002;97:3186-3194.

26. Baudet JS, Aguirre-Jaime A. The sedation increases the acceptance of repeat colonoscopies. Eur J Gastroenterol Hepatol 2012;24:775-780.

27. Aisenberg J, Brill JV, Ladabaum U, Cohen LB. Sedation for gastrointestinal endoscopy: new practices, new economics. Am J Gastroenterol 2005;100:996-1000.

28. Liu H, Waxman DA, Main R, Mattke S. Utilization of anesthesia services during outpatient endoscopies and colonoscopies and associated spending in 2003-2009. JAMA 2012;307:1178-1184.

29. Cohen LB. Sedation issues in quality colonoscopy. Gastrointest Endosc Clin N Am 2010;20:615-627.

30. Khiani VS, Soulos P, Gancayco J, Gross CP. Anesthesiologist involvement in screening colonoscopy: temporal trends and cost implications in the medicare population. Clin Gastroenterol Hepatol 2012;10:58-64.

31. Hassan C, Rex DK, Cooper GS, Benamouzig R. Endoscopistdirected propofol administration versus anesthesiologist assistance for colorectal cancer screening: a cost-effectiveness analysis. Endoscopy 2012;44:456-464.

32. Cai SR, Zhang SZ, Zhu HH, Zheng S. Barriers to colorectal cancer screening: a case-control study. World J Gastroenterol 2009;15:25312536.

33. Green AR, Peters-Lewis A, Percac-Lima S, et al. Barriers to screening colonoscopy for low-income Latino and white patients in an urban community health center. J Gen Intern Med 2008;23:834-840.

34. Vargo JJ, DeLegge MH, Feld AD, et al. Multisociety sedation curriculum for gastrointestinal endoscopy. Gastroenterology 2012;143:e18-e41.

35. Dumonceau J, Riphaus A, Beilenhoff U, et al. European curriculum for sedation training in gastrointestinal endoscopy: position statement of the European Society of Gastrointestinal Endoscopy (ESGE) and. Endoscopy 2013;45:496-504. 